\title{
The hard X-ray view of the low-luminosity blazar in the radio galaxy NGC 6251
}

\author{
M. Guainazzi ${ }^{1}$, P. Grandi ${ }^{2}$, A. Comastri ${ }^{3}$, and G. Matt ${ }^{4}$ \\ 1 XMM-Newton Science Operations Center, VILSPA, ESA, Apartado 50727, 28080 Madrid, Spain \\ e-mail: mguainaz@xmm.vilspa.esa.es \\ 2 Istituto di Astrofisica Spaziale e Fisica Cosmica (IASF-C.N.R.), Sezione di Bologna, via Gobetti 101, 40129 Bologna, Italy \\ 3 I.N.A.F., Osservatorio Astronomico di Bologna, via Ranzani 1, 40127 Bologna, Italy \\ 4 Dipartimento di Fisica, Università degli Studi "Roma Tre”, via della Vasca Navale 84, 00146 Roma, Italy \\ Received 21 February 2003 / Accepted 31 July 2003
}

\begin{abstract}
We present results from a BeppoSAX (July 2001) observation of the FR I radio galaxy NGC 6251, together with a re-analysis of archival ASCA (October 1994) and Chandra (September 2000) data. The weak detection above $10 \mathrm{keV}$ and the lack of iron fluorescent $\mathrm{K}_{\alpha}$ emission lines in the BeppoSAX spectrum rule out that the bulk of the X-ray emission is due to an obscured Seyfert nucleus. The study of the multiwavelength spectral energy distribution suggests instead that X-rays probably originate as inverse-Compton of synchrotron seed photons in a relativistic jet, indicating that NGC 6251 hosts a low radio luminosity $\left(L_{5 \mathrm{GHz}} \sim 10^{40} \mathrm{erg} \mathrm{s}^{-1}\right)$ blazar. The BeppoSAX spectrum is flatter than in the earlier ASCA observation. This might be due to the emergence of a different spectral component during phases of lower X-ray flux. In this context, we discuss some possible explanations for the intense and mildly-ionized fluorescent iron line measured by ASCA.
\end{abstract}

Key words. galaxies: active - galaxies: individual: NGC 6251 - galaxies: jets - galaxies: nuclei - X-rays: galaxies

\section{Introduction}

NGC 6251 is an E2 nearby $\left(z=0.02488\right.$; if $H_{0}=$ $70 \mathrm{~km} \mathrm{~s}^{-1} \mathrm{Mpc}^{-1}$, as assumed in this paper, $1^{\prime \prime}$ corresponds to about $500 \mathrm{pc}$ ) radio galaxy with a Faranoff-Riley I morphology, known to host a giant radio jet (Wagget et al. 1977), a Seyfert 2 nucleus (Werner et al. 2002), and almost edgeon dust lanes (Nieto et al. 1983). The nucleus is likely to contain a supermassive black hole with mass $M \sim 4-8 \times$ $10^{8} M_{\odot}$ (Ferrarese \& Ford 1999), as suggested by the presence of nuclear gas and a dust disc on scales of a few hundreds parsecs. NGC 6251 belongs to the outskirts of the cluster $\mathrm{Zw} 1609.0+8212$ (Young et al. 1979), whose influence on the properties of the galaxy should be, however, marginal (Prestage \& Peacock 1988).

The NGC 6251 jet is one of the most spectacular radio objects of the whole sky. It is a 4.5 long, highly collimated (opening angle $7.4^{\circ}$ ) structure (Perley et al. 1984). Regions of enhanced radio emission along the jet were labeled by Birkinshaw \& Worrall (1993) in the $330 \mathrm{MHz}$ radio map as A (10-40" from the nucleus), B (40-126"; actually structured in smaller sub-structures) and D (178-264", where the jet bends towards the North; it is as well highly structured). Between B and $\mathrm{D}$ one finds a region of low radio brightness (C). None of these region was observed to be a significant source of X-ray emission by ROSAT (Birkinshaw \& Worrall 1993). A sub-pc

Send offprint requests to: M. Guainazzi,

e-mail: mguainaz@scmm.vilspa.esa.es counter-jet was discovered only recently with Very Long Baseline Interferomer observations (Sudou et al. 2001). $U$ band Hubble Space Telescope images unveiled a region of extended emission, lying nearly perpendicular to the radio jet axis and the dust ring (Crane \& Vernet 1997), which is likely to originate from scattering of a nuclear continuum source.

Discovered in the X-rays by the Einstein Imaging Proportional Counter (Jones et al. 1986), NGC 6251 showed in the ROSAT PSPC observation (Birkinshaw \& Worrall 1993) an unresolved core $\left(F W H M<4^{\prime \prime}\right)$, with a possible extended halo on scales $\lesssim 100 \mathrm{kpc}$ (Mack et al. 1997). The first observation of NGC 6251 covering the intermediate X-rays (i.e.: $2-10 \mathrm{keV}$ ) was performed by ASCA. The presence of a bright $\mathrm{K}_{\alpha}$ fluorescence iron from ionized iron (centroid energy, $E_{\mathrm{c}} \simeq 6.68 \mathrm{keV}$; Equivalent Width, $E W \simeq 600 \mathrm{eV}$; Turner et al. 1997a; Sambruna et al. 1999), and the fact that the continuum could be best fit with a combination of a standard AGN power-law component (photon index, $\Gamma$, of 2.11) plus a thermal soft excess, suggested that ionized gas may significantly contribute in this energy band. Electron scattering of an otherwise invisible nuclear continuum was an interesting possibility, in light of the HST discovery of extended ionized gas, which could potentially act as a nuclear mirror ("warm mirror" hereinafter). Recently, an association has been proposed between NGC 6251 and the EGRET source 1EGJ 1621+8203 (Mukherjee et al. 2002). If this is confirmed, NGC 6251 would be one of the three radio galaxies - alongside with Cen A (Sreekumar et al. 1999) and 3EG J1735-1500 
(Combi et al. 2003) - detected in high-energy $\gamma$-rays. No detection by the Extreme Ultraviolet Explorer is reported in the literature (Marshall et al. 1995).

The deep BeppoSAX observation, described in this paper, aimed at verifying the interpretation scenario emerging from the ASCA outcomes. Thanks to its unsurpassed sensitivity in hard X-rays (i.e.: >10 keV; Boella et al. 1997), BeppoSAX would be easily able to detect a transmitted nuclear component piercing through a Compton-thick absorber (Matt et al. 2000). The results of this observation are presented in this paper, together with an analysis of archival ASCA and Chandra data of the same source. The log of the observations discussed in this paper is presented in Table 1.

While this paper was in the process of being accepted, we became aware of a recently accepted paper by Chiaberge et al. (2003), who discuss the same X-ray observations, together with literature and HST data. Their X-ray results are largely coincident with ours.

\section{BeppoSAX results}

\subsection{Data reduction}

BeppoSAX data were reduced according to standard procedures as in, e.g., Guainazzi et al. (1999). Scientific products for the imaging Low Energy Concentrator Spectrometer (LECS, Parmar et al. 1997, 0.5-4 keV) and Medium Energy Concentrator Spectrometer (MECS, Boella et al. 1997b, 1.8$10.5 \mathrm{keV}$ ) were extracted from circular regions of $8^{\prime}$ and $4^{\prime}$, respectively. Background spectra were extracted from blank sky field event lists provided by the BeppoSAX Science Data Center, and appropriate for the date of the observation. Products for the Phoswitch Detector System (PDS, Frontera et al. 1997, 13-200 keV) were extracted by plain subtraction of those corresponding to intervals when the instrument was observing NGC 6251 , and a region $\pm 3.5^{\circ}$ aside, according to the standard 96 s cycle.

All the spectra employed in this paper have been rebinned in order to oversample the intrinsic energy resolution of the instruments by a factor not larger than 3 , and to have a number of counts in each spectral channel higher than 30, in order to ensure the applicability of the $\chi^{2}$ test. In this paper: energies are quoted in the source rest frame; uncertainties on the spectral parameters are quoted at the $90 \%$ confidence level for one interesting parameter; uncertainties on the count rates are at $1 \sigma$ level, unless otherwise specified.

\subsection{Spectral results}

In Fig. 1 the results of the fit of the NGC 6251 BeppoSAX spectrum with a simple absorbed power-law is shown. The fit is statistically acceptable $\left(\chi^{2}=96.7 / 92\right.$ degrees of freedom, d.o.f.). The best-fit parameters and results are reported in Table 2. The PDS data points lay actually above the bestfit model, which is mainly determined by the statistics of the imaging instruments. The excess is, however, not highly significant. The ratio between the PDS flux and the extrapolation of the best fit model in the LECS/MECS energy band is $1.4 \pm 0.6$,

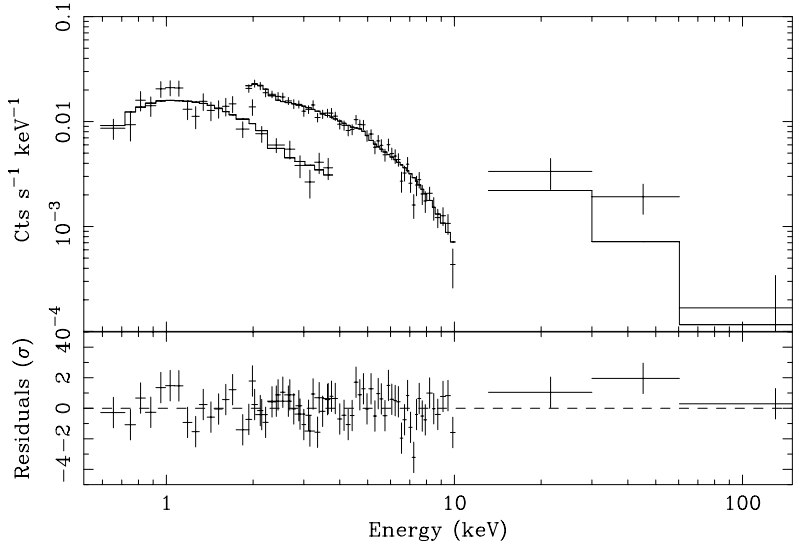

Fig. 1. BeppoSAX spectrum (upper panel) and residuals in units of standard deviations (lower panel), when the a photoelectrically absorbed power-law model is applied.

therefore only marginally exceeding the typical values of the cross-normalization factors between the PDS and the MECS (0.80-0.85; Fiore et al. 1998). Not surprisingly, several models provide comparably good descriptions of this hard excess, but none of them is required from the statistical point of view. A broken power-law, for instance, yields a $\Delta \chi^{2}=3.4$ for a reduction of the number of degrees of freedom $(\Delta v)$ by 2 (this quantity will be indicated as $\Delta \chi^{2} / \Delta v$ hereinafter), corresponding to a confidence level $\simeq 83.0 \%$

The BeppoSAX large source spectrum extraction region encompasses the putative ROSAT extended emission. We therefore tried to add a soft X-ray component, modeled by a collisionally ionized plasma (we used the mekal implementation in XSPEC V11.0 throughout this paper; Mewe et al. 1985; Liedahl et al. 1995; Arnaud \& Raymond 1992). Again, the improvement in the quality of the fit is not significant $\left(\Delta \chi^{2} / \Delta v=\right.$ $4.4 / 2)$. Consequently, the temperature is rather poorly determined $\left(k T=1.4 \pm_{0.7}^{0.9}\right)$. The $0.5-2.4 \mathrm{keV}$ flux of this component would be $(1.3 \pm 0.7) \times 10^{-13} \mathrm{erg} \mathrm{cm}^{-2} \mathrm{~s}^{-1}$, corresponding to about $15 \%$ of the non-thermal component in the same energy band. This model requires an absorbing column density slightly in excess with respect to the Galactic contribution along the line-of-sight to NGC $6251\left(5.7 \times 10^{20} \mathrm{~cm}^{-2}\right.$, Murphy et al. 1996).

No systematic residual feature is present at the energies, where $\mathrm{K}_{\alpha}$ fluorescence transitions of iron are expected. Negligible improvements of the $\chi^{2}$ are yielded by the addition of a narrow (i.e., intrinsic width, $\sigma=0$ ) Gaussian profile to the power-law model. $90 \%$ upper limits on the intensity of a neutral $(6.4 \mathrm{keV})$ or He-like $(6.7 \mathrm{keV})$ iron lines are 8.5 and $3.0 \times 10^{-6}$ photons $\mathrm{cm}^{-2} \mathrm{~s}^{-1}$, respectively, corresponding to $E W \mathrm{~s}$ of 160 and $60 \mathrm{eV}$, respectively. These upper limits are largely inconsistent with the ASCA detection (Turner et al. 1997a; cf. also Sect. 3.1 later). Simulations show that a $600 \mathrm{eV} \mathrm{EW}$ line would have been detected at the $6-7 \sigma$ confidence level in the MECS spectrum.

The $0.5-10 \mathrm{keV}$ flux during the BeppoSAX observation was $7.5 \times 10^{-12} \mathrm{erg} \mathrm{cm}^{-2} \mathrm{~s}^{-1}$, corresponding to un unabsorbed rest-frame luminosity of $1.13 \times 10^{43} \mathrm{erg} \mathrm{s}^{-1}$ in the same energy band. 
Table 1. Log of the observations presented in this paper.

\begin{tabular}{lccc}
\hline \hline Satellite & Start time & $\begin{array}{c}\text { Exposure time } \\
(\mathrm{ks})\end{array}$ & $\begin{array}{c}\text { Count rate } \\
\left(10^{-2} \mathrm{~s}^{-1}\right)\end{array}$ \\
\hline ASCA & 28-Oct.-1994 & $36^{a} / 44^{b}$ & $7.2 \pm 0.2^{a} / 3.63 \pm 0.13^{b}$ \\
Chandra/ACIS-I & 11-Sep.-2000 & 25 & $8.1 \pm 0.2$ \\
BeppoSAX & 19-Jul.-2001 & $28^{c} / 80^{d} / 72^{e}$ & $2.78 \pm 0.12^{c} / 5.55 \pm 0.09^{d} / 13 \pm 4^{e}$ \\
\hline
\end{tabular}

${ }^{a} \mathrm{SIS} 0,{ }^{b} \mathrm{GIS} 2,{ }^{c} \mathrm{LECS},{ }^{d} \mathrm{MECS},{ }^{e} \mathrm{PDS}$.

Table 2. Best-fit parameters and results for the analysis of the nuclear spectra of NGC 6251. Model legenda: $\mathrm{PL}=$ power-law; TH $=$ thermal component (mekal implementation in XSPEC) with solar abundances; GA = Gaussian emission line; BKPL = broken power-law.

\begin{tabular}{|c|c|c|c|c|c|c|c|c|}
\hline $\begin{array}{l}\text { Model } \\
\text { Mission }\end{array}$ & $\begin{array}{c}N_{\mathrm{H}} \\
\left(10^{21} \mathrm{~cm}^{-2}\right)\end{array}$ & $\Gamma$ & $\begin{array}{c}k T_{1} / \Gamma_{\text {soft }} \\
(\mathrm{keV}) /\end{array}$ & $\begin{array}{c}k T_{2} / E_{\text {break }} \\
(\mathrm{keV})\end{array}$ & $\begin{array}{c}E_{\mathrm{c}} \\
(\mathrm{keV})\end{array}$ & $\begin{array}{c}I_{\mathrm{c}} \\
\left(10^{-6} \mathrm{ph} \mathrm{cm}^{-2} \mathrm{~s}^{-1}\right)\end{array}$ & $\begin{array}{l}E W \\
(\mathrm{eV})\end{array}$ & $\chi^{2} /$ d.o.f. \\
\hline \multicolumn{9}{|l|}{ BeppoSAX } \\
\hline PL & $0.8 \pm_{0.3}^{0.6}$ & $1.79 \pm_{0.07}^{0.06}$ & $\ldots$ & $\ldots$ & $\ldots$ & $\ldots$ & $\ldots$ & $96.7 / 92$ \\
\hline \multirow[t]{4}{*}{$\mathrm{PL}+\mathrm{TH}$} & $1.0 \pm_{0.05}^{0.12}$ & $1.70 \pm_{0.16}^{0.12}$ & $1.4 \pm_{0.7}^{0.9}$ & $\ldots$ & $6.4^{\dagger}$ & $<8.0$ & $<160$ & $92.3 / 90$ \\
\hline & & & & $\ldots$ & $6.59^{\dagger}$ & $<4.5$ & $<80$ & \\
\hline & & & & $\ldots$ & $6.7^{\dagger}$ & $<3.0$ & $<60$ & \\
\hline & & & & $\ldots$ & $6.96^{\dagger}$ & $<2.1$ & $<50$ & \\
\hline \multicolumn{9}{|l|}{$A S C A$} \\
\hline PL & $0.9 \pm_{0.3}^{0.4}$ & $2.06 \pm_{0.07}^{0.08}$ & $\ldots$ & $\ldots$ & $\ldots$ & $\ldots$ & $\ldots$ & $259.7 / 225$ \\
\hline $\mathrm{PL}+\mathrm{TH}$ & $1.6 \pm_{0.8}^{0.7}$ & $2.00 \pm_{0.07}^{0.08}$ & $0.85 \pm_{0.15}^{0.34}$ & $\ldots$ & $\ldots$ & $\ldots$ & $\ldots$ & $240.6 / 223$ \\
\hline $\mathrm{PL}+2 \times \mathrm{TH}$ & $1.6 \pm_{0.8}^{2.1}$ & $2.3 \pm 0.4$ & $0.8 \pm_{0.2}^{0.3}$ & $6 \pm_{2}^{6}$ & $\ldots$ & $\ldots$ & $\ldots$ & $231.8 / 221$ \\
\hline $\mathrm{PL}+\mathrm{TH}+\mathrm{GA}$ & $1.7 \pm_{0.4}^{0.5}$ & $2.05 \pm_{0.10}^{0.11}$ & $0.84 \pm_{0.18}^{0.27}$ & & $6.59 \pm 0.16$ & $8 \pm 4$ & $500 \pm 200$ & $228.1 / 221$ \\
\hline $\mathrm{BKPL}+\mathrm{GA}$ & $4.0 \pm_{1.5}^{1.8}$ & $2.28 \pm_{0.14}^{0.17}$ & $3.7 \pm_{0.9}^{1.2}$ & $1.38 \pm_{0.09}^{0.15}$ & $6.62 \pm_{0.18}^{0.12}$ & $10 \pm 4$ & $800 \pm 300$ & $245.0 / 226$ \\
\hline
\end{tabular}

${ }^{\dagger}$ Gaussian emission profiles added to the best-fit continuum. Centroid energies $E_{\mathrm{c}}$ are fixed. Upper limits are at the $90 \%$ confidence level.

\section{Comparison with previous X-ray observations}

\subsection{ASCA}

We have retrieved the data of the ASCA observation of NGC 6251 from the public archive as screened event lists. Spectra were extracted from regions of radii 4', 3'.1 and 3.75 in the SIS0, SIS1 (grade 0, 2, 3, 4) and GIS detectors, respectively (they therefore encompass as well the ROSAT extended emission region). Background spectra were extracted from regions in the field of view of the detectors, free from contaminating sources. Response matrices were generated with the packages included in the LHEASOFT v5.0 software. The spectral analysis was performed in the $0.5-10 \mathrm{keV}$ and $0.7-10 \mathrm{keV}$ energy bands, for the SIS and GIS instruments, respectively. The results of our analysis substantially coincide with those presented by Turner et al. (1997) and Sambruna et al. (1999), and we summarize them in this paper for the sake of clarity only.

A simple power-law is a fairly good representation of the ASCA spectra $\left(\chi^{2}=259.7 / 225\right.$ d.o.f. $)$. However, the addition of a mekal component improves significantly the quality of the fit $\left(\Delta \chi^{2} / \Delta v=19.1 / 2\right.$, corresponding to an F-test confidence level $\simeq 99.97 \%$ ). The addition of a multitemperature blackbody (model diskbb in XSPEC) yields, on the contrary, a negligible improvement to the quality of the fit. Modeling the continuum in terms of a broken power-law yields a comparatively worse fit as well. A systematic excess around the energy where fluorescent $\mathrm{K}_{\alpha}$ transitions from iron are expected

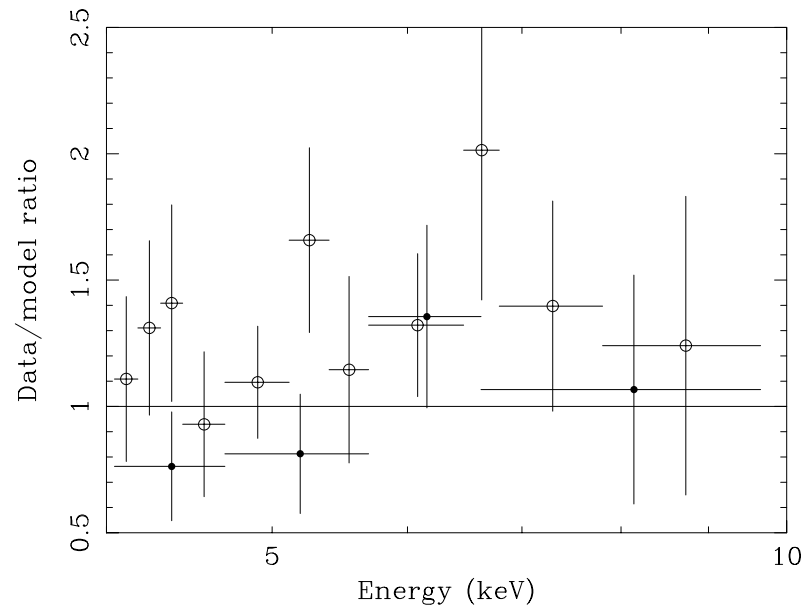

Fig. 2. GIS2 (filled dots) and GIS3 (open circles) residuals against a power-law plus optically thin thermal plasma continuum in the 4$10 \mathrm{keV}$ bands.

is observed (see Fig. 2). It can be cured with an unresolved Gaussian profile $\left(\Delta \chi^{2} / \Delta v=12.5 / 2\right.$, corresponding to a confidence level $\simeq 99.7 \%)$, or with a hotter $(k T \simeq 6 \mathrm{keV})$ thermal component $\left(\Delta \chi^{2} / \Delta v=8.8 / 2\right.$, corresponding to a confidence level $\simeq 98.6 \%$ ).

In the best-fit model (cf. Table 2$)$, the power-law index $(\Gamma \simeq$ $2.05)$ is significantly steeper than observed by BeppoSAX. The temperature of the colder thermal component is $k T \simeq 0.8 \mathrm{keV}$, and its $0.5-2.4 \mathrm{keV}$ flux $\left((1.6 \pm 0.4) \times 10^{-13} \mathrm{erg} \mathrm{cm}^{-2} \mathrm{~s}^{-1}\right)$ is 
in good agreement with that of the corresponding component in the BeppoSAX spectrum. The iron line centroid energy is consistent with $\mathrm{K}_{\alpha}$ fluorescence from mildly ionized to He-like iron. Its $E W$ is $500 \pm 200 \mathrm{eV}$, significantly larger than the upper limits measured by BeppoSAX. The difference is most likely due to a change in the underlying continuum, as the intensity of the ASCA line $\left((8 \pm 4) \times 10^{-6}\right.$ photons $\left.\mathrm{cm}^{-2} \mathrm{~s}^{-1}\right)$ is marginally consistent with the BeppoSAX upper limits. However, an intrinsic variability of the iron line cannot be ruled out.

The observed $0.5-10 \mathrm{keV}$ flux during the ASCA observation was $2.7 \times 10^{-12} \mathrm{erg} \mathrm{cm}^{-2} \mathrm{~s}^{-1}$, corresponding to an unabsorbed total luminosity of $4.4 \times 10^{42} \mathrm{erg} \mathrm{s}^{-1}$ in the same energy band.

\subsection{Chandra}

Data of an ACIS-I NGC 6251 Chandra observation were retrieved from the public archive. A bright source (count rate $\left.(8.1 \pm 0.2) \times 10^{-2} \mathrm{~s}^{-1}\right)$ is detected with coordinates: $\alpha_{2000}=$ $16^{\mathrm{h}} 32^{\mathrm{m}} 31^{\mathrm{s}} 8 ; \delta_{2000}=+82^{\mathrm{h}} 32^{\mathrm{m}} 16^{\mathrm{s}}$, i.e. $0.6^{\prime \prime}$ distant from the optical nucleus of NGC 6251, well within the accuracy of the Chandra attitude reconstruction. At this count rate level, a Chandra source is likely to be substantially affected by pile-up, given the instrumental mode employed (Time Exposure Mode with a $3.2 \mathrm{~s}$ frame time). We have tried to fit the $0.5-8 \mathrm{keV}$ spectrum extracted from the innermost $5^{\prime \prime}$ with a photoelectrically absorbed power-law, corrected for pile-up according to the XSPEC implementation of J. Davis' algorithm (model pileup in XsPEC; Arnaud \& Dorman 2002). The fit is acceptable $\left(\chi^{2}=57.1 / 78\right.$ d.o.f.), and yields best-fit parameters which are consistent with those measured during the BeppoSAX observation, save a $60 \%$ lower flux: $N_{\mathrm{H}}=(1.6 \pm 0.5) \times 10^{21} \mathrm{~cm}^{-2}$; $\Gamma=1.76 \pm 0.16 ; 0.5-10 \mathrm{keV}$ flux of $4.5 \times 10^{-12} \mathrm{erg} \mathrm{cm}^{-2} \mathrm{~s}^{-1}$.

Thanks to the unprecedented spatial resolution of the ACIS-S, the issue of the spatial extension can be better addressed. No evidence for extended emission along the arcminutes scale jet is detected, as the ROSAT observation had already shown in the soft X-rays only (Birkinshaw \& Worrall 1993). The upper limits on the X-ray fluxes of the regions A to D are reported in Table 3. They are by a factor 5 to 10 tighter than those determined by Birkinshaw \& Worrall (1993). However, the upper limit on the knot " $D$ " flux density is inconsistent with the detection $\left(S_{v}=13 \pm 2 \mathrm{nJy}\right.$ ) obtained by Mack et al. (1997) from a reanalysis of the same ROSAT/PSPC observation discussed by Birkinshaw \& Worrall (1993).

In the innermost $4^{\prime}$ around the NGC 6251 core only two sources, alongside with the nucleus itself, are detected at a signal-to-noise ratio higher than 3 . Their positions and count rates are reported in Table 4. None of them exhibits a clear association with any radio structures.

\section{Discussion}

\subsection{The nature of the nuclear $X$-ray emission in NGC 6251}

The main goal of the BeppoSAX observation described in this paper was to test whether the strong $(E W \sim 600 \mathrm{eV})$,

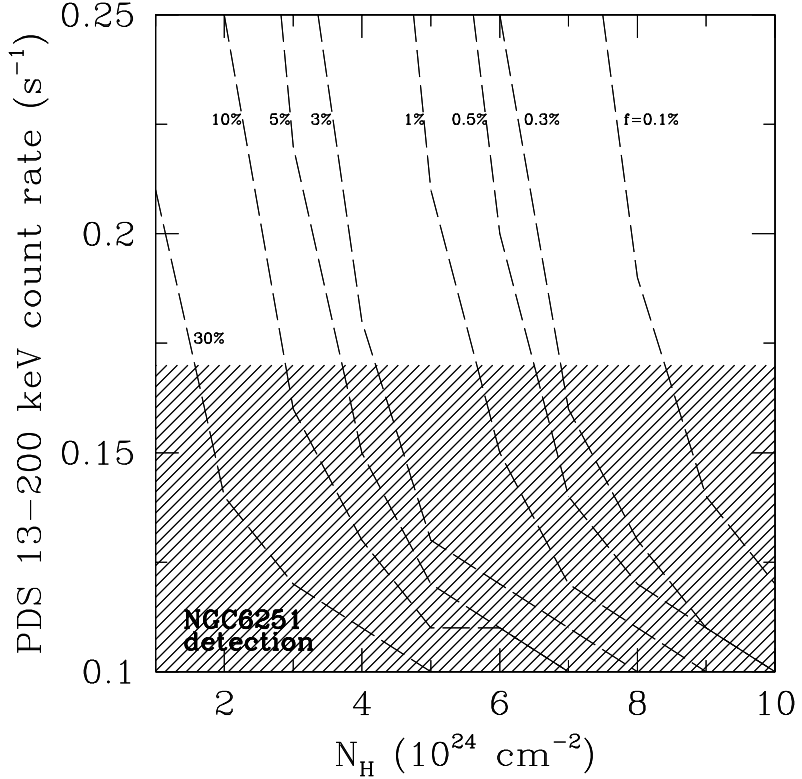

Fig. 3. PDS count rates in the $13-200 \mathrm{keV}$ energy band against the column density of a Compton-thick absorber covering the NGC 6251 nucleus, for different values of the scattering fraction $f$. The shaded area indicates the PDS detection yielded by the BeppoSAX observation of NGC 6251. $f$ is defined by the ratio between the normalizations of the transmitted and the warm scattered components, assuming an optically thin scatterer.

ionized $\mathrm{K}_{\alpha}$ fluorescent iron line observed in the ASCA spectrum (Turner et al. 1997a) was due to a "warm mirror" reflection-dominated, Compton-thick Seyfert 2 spectrum. In the light of the BeppoSAX observation outcomes, this possibility is rather unlikely. The PDS detection $\left(0.13 \pm 0.04 \mathrm{~s}^{-1}\right.$ in the $13-200 \mathrm{keV}$ energy band) is statistically consistent with the extrapolation of the $2-10 \mathrm{keV}$ spectrum. This constraints the column density of any Compton-thick absorber to the nucleus to be $\gtrsim 3 \times 10^{24}\left(6 \times 10^{24}\right) \mathrm{cm}^{-2}$ for a $10 \%$ (1\%) scattering fraction (see Fig. 3). Moreover, the $2-10 \mathrm{keV}$ BeppoSAX spectrum lacks any trace of fluorescence $\mathrm{K}_{\alpha}$ ionized iron lines, which are almost always observed in reflection-dominated Seyfert 2 galaxies (Turner et al. 1997b; Matt et al. 2000). The upper limits on their $E W \mathrm{~s}$ are rather strict, ranging between 60 and $80 \mathrm{eV}$ for He- and H-like iron. The standard AGN $2-10 \mathrm{keV}$ spectral index $\left(\Gamma \simeq 1.7\right.$ ), and the lack of $\mathrm{K}_{\alpha}$ neutral iron line (again, with a rather tight $E W$ upper limit: $160 \mathrm{eV}$ ) in the BeppoSAX spectrum rule out a significant contribution by Compton-reflection from the inner side of the molecular torus (Krolik et al. 1994; Ghisellini et al. 1994; Matt et al. 1996) In principle, the line flux could be suppressed by resonant trapping at the ionized surface of an accretion disk (Matt et al. 1993). However, further pieces of evidence rule out that the X-ray spectrum is dominated by reflection of an otherwise invisible Seyfert nucleus.

Some hints come from the study of the multiwavelength Spectral Energy Distribution (SED). Recently, Fossati et al. (1998) have proposed a unified scheme to explain the multivawelenght SED of blazars, whose properties (peak frequency of the synchrotron and inverse-Compton components, 
Table 3. $90 \%$ upper limits on the ACIS-I count rates $(C R), 0.5-$ 10 fluxes $(F)$, and $1 \mathrm{keV}$ flux density $\left(S_{v}\right)$ across the NGC 6251 jet regions (following their definition in Birkinshaw \& Worrall 1993). The distance $d$ is defined from the inner-outer border of each region to the core. Fluxes are calculated assuming a power-law spectrum, with $\Gamma=$ 2 and photoelectric absorption column density $N_{\mathrm{H}}=5.7 \times 10^{20} \mathrm{~cm}^{-2}$.

\begin{tabular}{lccccc}
\hline \hline Region & $\begin{array}{c}d \\
(\operatorname{arcsec})\end{array}$ & $\begin{array}{c}\text { Area } \\
\left(\operatorname{arcsec}^{2}\right)\end{array}$ & $\begin{array}{c}C R \\
\left(10^{-4}\right)\end{array}$ & $\begin{array}{c}F \\
a\end{array}$ & $\begin{array}{c}S_{v} \\
(\mathrm{nJy})\end{array}$ \\
\hline $\mathrm{A}$ & $10-40$ & 46.8 & 2.8 & 2.3 & 0.34 \\
$\mathrm{~B}$ & $40-126$ & 140.6 & 2.2 & 1.8 & 0.26 \\
$\mathrm{C}$ & $126-178$ & 78.1 & 2.9 & 2.4 & 0.36 \\
$\mathrm{D}$ & $178-264$ & 124.9 & 1.9 & 1.6 & 0.24 \\
\hline
\end{tabular}

${ }^{a}$ In the $0.5-10 \mathrm{keV}$ band, in units of $10^{-15} \mathrm{erg} \mathrm{cm}^{-2} \mathrm{~s}^{-1}$.

Table 4. Sources detected in the ACIS-I observation of the NGC 6251 field, within 4' from the core.

\begin{tabular}{cccc}
\hline \hline$\#$ & $\alpha_{2000}$ & $\delta_{2000}$ & $\begin{array}{c}\text { count rate } \\
\left(10^{-4} \mathrm{~s}^{-1}\right)\end{array}$ \\
\hline 1 & $16^{\mathrm{h}} 32^{\mathrm{m}} 31^{\mathrm{s}} .8$ & $+82^{\mathrm{h}} 32^{\mathrm{m}} 16^{\mathrm{s}}$ & $810 \pm 20^{a}$ \\
2 & $16^{\mathrm{h}} 33^{\mathrm{m}} 00 \mathrm{~s} .5$ & $+82^{\mathrm{h}} 31^{\mathrm{m}} 12^{\mathrm{s}}$ & $4.8 \pm 1.8$ \\
3 & $16^{\mathrm{h}} 33^{\mathrm{m}} 21^{\mathrm{s}} .3$ & $+82^{\mathrm{h}} 31^{\mathrm{m}} 56^{\mathrm{s}}$ & $5.6 \pm 1.8$ \\
\hline
\end{tabular}

${ }^{a}$ NGC 6251 nucleus.

luminosity ratio between them) are mainly governed by a single parameter related to the overall luminosity. This scheme can be applied to help identifying the nature of the bulk of the X-ray emission from the NGC 6251 nucleus. In Fig. 4 we show a color-color diagram between the radio $(v=6 \mathrm{~cm})$, soft X-ray $(0.5-4.5 \mathrm{keV})$, and infrared $(\lambda=25 \mu)$ luminosities. The dotted line in this diagram represents the trace followed by blazars according to the Fossati scheme. In the same plot the cross represents the position of the Seyfert 2 galaxies of the Mass-Hesse et al. sample (1995). The $6 \mathrm{~cm}$ radio luminosity of NGC 6251 is $(1.0 \pm 0.1) \times 10^{40} \mathrm{erg} \mathrm{s}^{-1}$ (Jones et al. 1986). The data points corresponding to the ASCA and BeppoSAX observations lay intriguingly well on the blazar track. This supports the idea that the bulk of the nuclear emission in NGC 6251 is due to a jet.

Chiaberge et al. (1999) discovered a clear correlation between the optical $\left(F_{\mathrm{O}}\right)$ and the radio core luminosity $\left(F_{\mathrm{R}}\right)$ in a sample of FRI galaxies extracted from the 3C catalogue and observed with the HST WFC2. Assuming the NGC 6251 SED published by Ho (1999), $\log \left(F_{\mathrm{R}} / F_{\mathrm{O}}\right) \simeq 3.4$, in perfect agreement with the value derived from the $3 \mathrm{C}$ correlation $(3.7 \pm 0.4$; the uncertainties represent the rms scattering of the data points in the correlation), and largely inconsistent with values typically measured in radio-loud quasars $(\simeq-1$; Elvis et al. 1994). This supports a common origin for the radio and optical emission as synchrotron radiation. HST observations in the $U$-band measured a rather high degree of polarization (close to $50 \%$ ) in clumps close to the nucleus along the radio axis. Crane \& Vernet (1997) suggest that the UV emission of these clumps is due to scattering. However, such an evidence is consistent as well with the possibility that the synchrotron-dominance extends well within the UV range.

We tried to fit the overall NGC 6251 SED from radio to $\gamma$-rays (X-rays represented by the BeppoSAX spectrum)

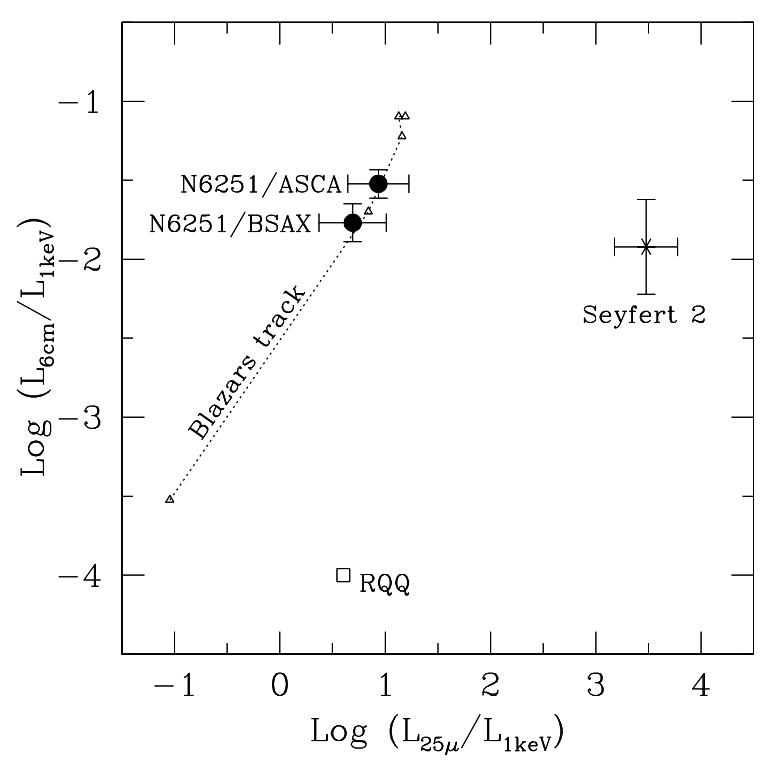

Fig. 4. Radio (6 cm), X-ray (0.5-4.5 keV) and IR (25 $\mu$ ) color-color diagram. The dotted line represents the blazar track according to the blazar unified scheme of Fossati et al. (1998). The filled circles represent the data points corresponding to the NGC 6251 ASCA and BeppoSAX observations. The cross corresponds to the Seyfert 2 sample of Mass-Hesse et al. (1995). The empty square represents the SED of radio-quiet quasars (Elvis et al. 1994).

with an homogeneous Synchrotron Self-Compton (SSC) model (Tavecchio et al. 1998). This model assumes that synchrotron radiation is produced by relativistic electrons with density $n_{\mathrm{e}}$ continuously injected in a spherical region of radius $r$ with a magnetic field $B$ and moving with bulk factor $\Gamma_{\text {bulk }}$ at an angle $\theta$ with respect to the line of sight (the Doppler factor is therefore $\left.\delta=\left[\Gamma_{\text {bulk }}(1-\beta \cos \theta)\right]^{-1}\right)$. These photons are subsequently upscattered by the same electrons. Following Tavecchio et al. (1998), the electron energy distribution is modeled with a broken power-law with indices $n_{1}<3$ and $n_{2}>3$ below and above a break energy $\gamma_{\mathrm{b}}$, respectively. The IRAS data points, although in principle available, have not been included in the fit due to the unknown contamination from the host galaxy. The best fit from radio to $\gamma$ frequencies (dashed line in Fig. 5) fails to reproduce the BeppoSAX spectral shape and slightly deviates below $10^{10} \mathrm{~Hz}$. If one forces the model to match the BeppoSAX data point (dotted line in Fig. 5), the radio emission is more overproduced. This may indicate that self-absorption (which is not explicitly included in SSC models) plays an important role redwards the synchrotron peak. Given the fact that the SED data points correspond to non-simultaneous observations, one cannot push too far the comparison between the model and the observation. Nonetheless, the overall properties of the best-fit SSC models are not strongly dependent on the details of the fit. The synchrotron peak is below $10^{14} \mathrm{~Hz}$, whereas the Compton peak remains constrained between $10^{21.5}$ and $10^{22.5} \mathrm{~Hz}$. As a reference the best-fit parameters for the fits are in the range: $B=0.01-0.15 \mathrm{G}, \delta=2-4, n_{1}=1.75-2.6, n_{2}=4.1-5.4$, $\gamma_{\mathrm{b}}=6.3 \times 10^{3}-1.6 \times 10^{4} n_{\mathrm{e}}=10^{5}-10^{6} \mathrm{~cm}^{-3}$, and $r=1-$ $8 \times 10^{16} \mathrm{~cm}$. The reader is referred to Chiaberge et al. (2003) 


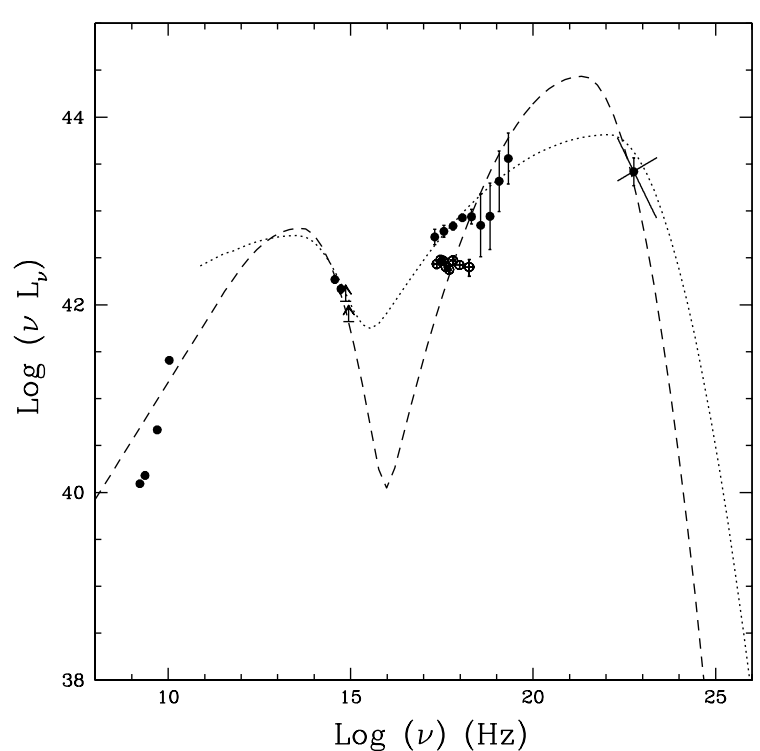

Fig. 5. NGC 6251 SED. Data are from not-simultaneous observations compiled by Ho (1999), save the and X-ray data (BeppoSAX, dots; this paper), and the putative EGRET detection. The dashed line represents the best radio- $\gamma$ fit SSC model; the dotted line represents the fit with an SSC model, forced to reproduce the BeppoSAX spectral shape. The ASCA spectrum (empty circles) is shown as well for reference.

for a more detailed comparison between the NGC 6251 SED and SSC models.

It is hard to interpret the spectral variability between the ASCA (empty circles in Fig. 5) and the BeppoSAX spectra in terms of pure SSC model. In principle, the ASCA spectrum may represent the trailing edge of the synchrotron component. However, this would imply a shift of the synchrotron peak by more than 4 orders of magnitude, which is rather unlikely. Alternatively, the steeper ASCA spectrum may imply that a different spectral component may be emerging during phases of low X-ray flux. This component may be completely outshined during BeppoSAX-like, X-ray brighter states. We will further discuss this possibility in Sect. 4.2, in connection with the strong fluorescent iron line observed in the ASCA spectra only.

Recently, it has been suggested that the bulk of the $\mathrm{X}$-ray emission in radio galaxies may be due to a hot inner accretion flow, following a line of thought suggested more than 20 years ago by Rees et al. (1982). Ho (1999) estimates the bolometric luminosity of NGC 6251 as $\sim 10^{-4} L_{\text {Edd }}$. This may indicate that an Advection Dominated Accretion Flow (ADAF; Narayan \& Yi 1995) is responsible for the bulk of the emission in the NGC 6251 core. In this scenario, X-rays are mainly produced via bremsstrahlung by a distribution of thermal electrons with typical temperatures $k T \sim 100 \mathrm{keV}$ (Di Matteo et al. 2000). In Fig. 6 we compare the ratio between the $V$ and the $1 \mathrm{keV}$ flux density (BeppoSAX measurement) in NGC 6251 with the values expected by the blazar unification scenario (Fossati et al. 1998) and by models of ADAF applied to the multiwavelength SED of nearby elliptical galaxies suspected to host low radiative efficiency accretion flows (Di Matteo et al. 2000; we consider hereby models

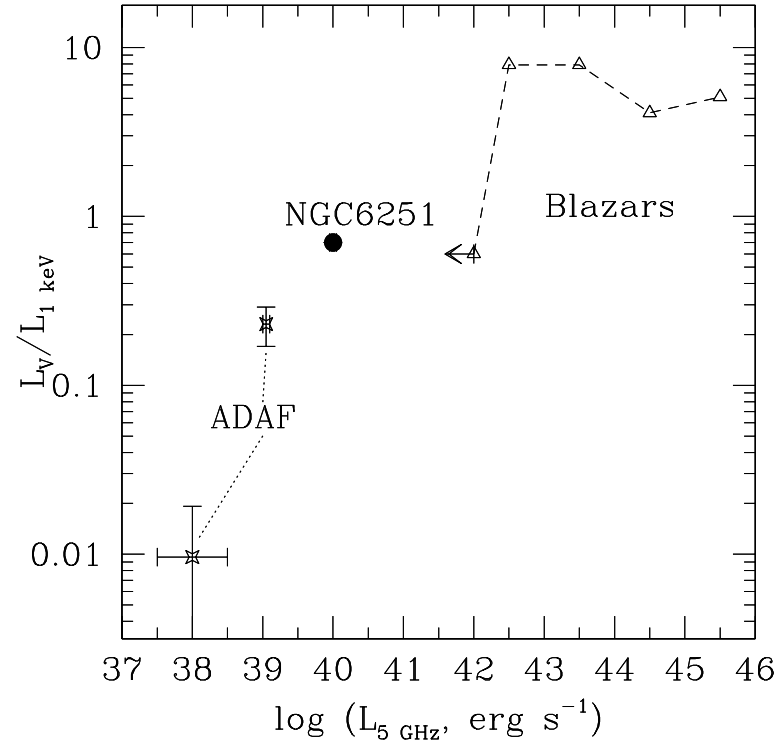

Fig. 6. Radio between the $1 \mathrm{keV}$ and the $V$-band flux density in: a) NGC 6251 (filled circle); b) the blazar radio luminosity classes in the unified scenario after Fossati et al. (1998); c) ADAF models applied to a sample of nearby elliptical galaxies (Di Matteo et al. 2000; details in text). In the last case, the error bars on the $y$-axis represent the rms of the sample values in the corresponding radio luminosity interval.

with wind, and outermost hot accretion radius of 300 gravitational radii). The NGC 6251 data point is in principle consistent with both scenarios. Applying a bremsstrahlung model to the BeppoSAX spectrum, one gets indeed a total X-ray luminosity $L_{\mathrm{X}} / L_{\mathrm{Edd}}=(2 \pm 1) \times 10^{-3}$. However, the fit is significantly worse than with a simple power-law $\left(\chi^{2}=110.0 / 92\right.$ d.o.f. $)$. A composite bremsstrahlung and power-law fit (the latter component taking into account possible Comptonization effects) yields an implausibly low electron temperature $(k T \simeq 3 \mathrm{keV})$. These pieces of evidence are in agreement with the conclusions drawn by Ferrarese \& Ford (1999), who remark that the $n u$ clear non-thermal bolometric optical luminosity in NGC 6251 is larger than predicted for accretion at the Bondi rate with $10 \%$ efficiency, making the case for an ADAF far less compelling.

\subsection{Additional components in the nuclear $X$-ray spectrum of NGC 6251}

As already noticed by Turner et al. (1997), a soft X-ray excess is observed in the NGC 6251 ASCA spectra. A multitemperature blackbody is not able to fit the excess, arguing against the possibility that this component originates in an accretion disk. The best-fit temperature of the soft excess, if modeled with an optically thin, collisionally excited plasma, is $k T \simeq 0.8 \mathrm{keV}$, with an unabsorbed luminosity of $\simeq 3.6 \times 10^{41} \mathrm{erg} \mathrm{s}^{-1}$. Such a temperature is typical of gaseous halos in elliptical galaxies (Matsumoto et al. 1997). The parameters of this component are consistent with those of a similar component in the BeppoSAX spectrum, which is, however, not required from the statistical point of view. The ASCA temperature is in turn consistent, within the rather large statistical uncertainties, with the temperature of the extended halos measured by the 
Einstein/IPC and the ROSAT/PSPC in NGC 6251 (for the latter, $k T=0.3-0.8 \mathrm{keV}$; Birkinshaw \& Worrall 1993 and references therein). Similarly, the $0.5-2.4 \mathrm{keV}$ unabsorbed fluxes of this component measured by ASCA and BeppoSAX are consistent with the ROSAT/PSPC soft X-ray "halo" flux, integrated across its whole extension $\left(\left(2.4 \pm_{2.0}^{4.2}\right) \times 10^{-13} \mathrm{erg} \mathrm{cm}^{-2} \mathrm{~s}^{-1}\right.$; Mack et al. 1997). It is therefore straightforward to identify the soft excess in the large ASCA aperture with the diffuse emission in ROSAT. Mack et al. (1997) discuss (and rule out) the possibility that such a plasma could be the confining medium for the jet, along the whole structure up to knot "D". A $\simeq 10^{7} \mathrm{~K}$ thermal emission responsible for the confinement of the jet in NGC 6251 should not extend beyond scales larger than $60 \mathrm{pc}$. On such small scales, it might be marginally resolvable by Chandra. Unfortunately, the pile-up affecting the Chandra observation prevented us from deriving precious constraints on the X-ray extension around the NGC 6251 core.

The ASCA spectrum unveils the possible presence of a third spectral component, whose main signature is a bright $(E W \simeq 600) \mathrm{eV}$ and significantly ionized $\mathrm{K}_{\alpha}$ fluorescence iron line. This component, even if present with comparable flux in the BeppoSAX observation, would have easily missed detection, due to the fact that the BeppoSAX non-thermal continuum $6 \mathrm{keV}$ flux density was $\geq 6$ times larger than in ASCA. This "third" component cannot be produced by the superposition of unresolved discrete sources, integrated in the large ASCA aperture. The Chandra image shows only two sources in the innermost $4^{\prime}$ around the NGC 6251 core, whose total count rate $\left[(1.1 \pm 0.3) \times 10^{-3}\right]$, corresponds to a $0.5-9 \mathrm{keV}$ flux $\simeq 1.0 \times 10^{-14} \mathrm{erg} \mathrm{cm}^{-2} \mathrm{~s}^{-1}$ (assuming a thermal plasma with $k T=6 \mathrm{keV}$ ). This is more than one order of magnitude less than the flux of the ASCA component in the same energy band $\left(2 \times 10^{-13} \mathrm{erg} \mathrm{cm}^{-2} \mathrm{~s}^{-1}\right)$. This component may therefore represent the "tip of the iceberg" of an underlying Seyfert nuclear "warm scattering" (Matt et al. 1996, 2001), whose relative contribution becomes not-negligible during phases of lower blazar activity. Alternatively, this component may represent a hotter and weaker phase of the thermal emission. It has already been pointed out that the kpc-scale jet structure in NGC 6251 requires an ambient gas with temperatures of $2-5 \mathrm{keV}$ to confine it. So far, the lack of detection of a gas component with such an high-temperature had led to the problem of an "overpressurized" jet in NGC 6251. It would be tempting to speculate that the high-temperature thermal component measured by ASCA is the required "missing link" to ensure the thermodynamical stability of the kpc-scale jet. Unfortunately, only rather strict upper limits can be set on any X-ray emission associated with the radio jet (see Table 3). Alternatively, the line could be produced in the interaction between the "bloated" base of a jet with a stratified velocity structure and the circumnuclear matter, as proposed by Chiaberge et al. (2000) in the context of a possible unification model between BL Lac objects and FR I radio galaxies. The possibility that the iron line is associated with the highly ionized "skin" of an accretion disk cannot be in principle ruled out, although evidence for significantly ionized disk lines is rather scanty so far.

All the best-fit X-ray models discussed in this paper require an amount of cold photoelectric absorption in excess to the Galactic contribution. The weighted between the BeppoSAX and ASCA measurements is $N_{\mathrm{H}}=(1.06 \pm 0.11) \times$ $10^{21} \mathrm{~cm}^{-2}$. This is in excellent agreement with the column density through the dusty disk, as derived from its visual extinction $\left(A_{V}=0.61 \pm 0.12\right.$; Ferrarese \& Ford 1999), if standard gas-todust Galactic ratios are assumed. This is in agreement with the idea that the standard pc-scale optically and geometrically thick torus - even if present in FRI low-luminosity radio galaxies - does not intercept our line-of-sight to the nucleus (Chiaberge et al. 2002).

Acknowledgements. This paper benefitted of the stimulating scientific environment at the Workshop "AGN spectroscopy with Chandra and XMM-Newton", held at the Max Planck Institut für Extraterrestrische Physik in Garching. Support by Maria Teresa Fiocchi in using the SSC models fitting facility at the A.S.I. Science Data Center is gratefully acknowledged. Last, but not least, comments by the referee (Dr. M. Boettcher) greatly helped us to sharpen the focus of the discussion.

\section{References}

Arnaud, K., \& Dorman, B. 2002, XSPEC User Manual v11.0

Arnaud, M., \& Raymond, J. 1992, ApJ, 398, 394

Birkinshaw, M., \& Worrall, D. M. 1993, ApJ, 412, 568

Boella, G., Butler, R. C., Perola, G. C., et al. 1997, A\&AS, 122, 299

Boella, G., Chiappetti, L., Conti, G., et al. 1997, A\&AS, 122, 327

Chiaberge, M., Capetti, A., \& Celotti, A. 1999, A\&A, 349, 77

Chiaberge, M., Celotti, A., Capetti, A., \& Ghisellini, G. 2000, A\&A, 358,104

Chiaberge, M., Duccio Macchetto, F., Sparks, W. B., et al. 2002, ApJ, 571,247

Chiaberge, M., Gilli, R., Capetti, A., Duccio \& Macchetto, F. 2003, ApJ, in press [astro-ph/0307120]

Combi, J. A., Romero, C. E., Paredes, J. M., Torres, D. F., \& Ribó, M. 2003, ApJ, 588, 731

Crane, P., \& Vernet, J. 1997, ApJ, 486, L91

Di Matteo, T., Quataert, E., Allen, S. W., Narayan, R., \& Fabian, A. C. 2000, MNRAS, 311, 507

Elvis, M., Wilkes, B. J., McDowell, J. C., et al. 1994, ApJS, 95, 1

Ferrarese, L., \& Ford, H. C. 1999, ApJ, 515, 583

Fiore, F., Guainazzi, M., \& Grandi, P. 1998, Cookbook of BeppoSAX data analysis (Roma: BeppoSAX SDC)

Fossati, G., Maraschi, L., Celotti, A., Comastri, A., \& Ghisellini, G. 1998, MNRAS, 299, 433

Frontera, F., Costa, E., dal Fiume, D., et al. 1997, A\&AS, 122, 347

Ghisellini, G., Haardt, F., \& Matt, G. 1994, MNRAS, 267, 743

Guainazzi, M., Perola, G. C., Matt, G., et al. 1999, A\&A, 346, 407

Ho, L. C. 1999, ApJ, 516, 672

Krolik, J. H., Madau, P., \& Życki, P. T. 1994, ApJ, 420, L57

Jones, D. L., Unwin, S. C., Readhead, A. C. S., et al. 1986, ApJ, 305, 684

Liedahl, D. A., Osterheld, A. L., \& Goldstein, W. H. 1985, ApJ, 438, L115

Mack, K. H., Kerp, J., \& Klein, U. 1997, A\&A, 324, 870

Marshall, H. L., Fruscione, A., \& Carone, T. E. 1995, ApJ, 439, 90

Matsumoto, H., Koyama, K., Awaki, H., et al. 1997, ApJ, 482, 133

Matt, G., Fabian, A. C., \& Ross, R. R. 1993, MNRAS, 261, 346

Matt, G., Brandt, W. N., \& Fabian, A. C. 1996, MNRAS, 280, 823

Matt, G., Fabian, A. C., Guainazzi, M., et al. 2000, MNRAS, 318, 173

Matt, G., Guainazzi, M., Perola, G. C., et al. 2001, A\&A, 377, L31

Mewe, R., Gronenschild, E. H. B. M., \& van der Oord, G. H. J. 1985, A\&AS, 62, 197 
Mukherjee, R., Halpern, J., Mirabal, N., \& Gotthelf, E. V. 2002, ApJ, Sreekumar, P., Bertsch, D. L., Hartman, R. C., Nolan, P. L., \& 574,693

Thompson, D. J. 1999, Astropar. Physics, 11, 221

Murphy, E. M., Lockman, F. J., Laor, A., \& Elvis, M. 1996, ApJS, Sudou, H., Taniguchi, Y., Ohyama, Y., et al. 2001, PASJ, 52, 989 105,369

Narayan, R., \& Yi, I. 1995, ApJ, 444, 231

Nieto, J. C., Coupinot, G., LeLievre, G., \& Madsen, C. 1983, MNRAS, 203, 39P

Parmar, A. N., Martin, D. D. E., Bavdaz, M., et al. 1997, A\&AS, 122, 309

Perley, R. A., Bridle, A. H., \& Willis, A. G. 1984, ApJS, 54, 291

Prestage, R. M., \& Peacock, J. A. 1988, MNRAS, 230, 131

Rees, M.J., Begelman, M. C., Blandford, R. D., \& Phinney, E. S. 1982, Nature, 295, 17

Sambruna, R., Eracleous, M., \& Mushotzky, R. 1999, ApJ, 526, 60

Turner, T. J., Garaschi, L., \&hiserlini, G. 1998, ApJ, 509, 608 ApJS, 113, 23

Turner, T. J., George, I. M., Nandra, K., \& Mushotzky, R. F. 1997b, ApJ, 488, 164

Wagget, P. C., Warner, P. J., \& Baldwin, J. E. 1977, MNRAS, 181, 465 Werner, P. M., Worrall, D. M., \& Birkinshaw, M. 2000, MNRAS, 317, 105

White, R. L., \& Becker, R. H. 1992, ApJS, 79, 331

Young, P. J., Sargent, W. L. W., Kristian, J., \& Westphal, J. A. 1979, ApJ, 234, 76 